\title{
Multidrug resistance behaviors of clinical Pseudomonas aeruginosa strains associated to pigment coloration
}

Ashish Kothari ${ }^{1}$, Rohitash Yadav ${ }^{2}$, Prashant Rajput ${ }^{3}$, Vanya Singh ${ }^{1}$, Karanvir Kaushal ${ }^{3}$, Neeraj Jain $^{\star 4,5}$ and Balram Ji Omar*1

${ }^{1}$ Department of Microbiology, All India Institute of Medical Sciences, Rishikesh-249203, India; ${ }^{2}$ Department of Pharmacology, All India Institute of Medical Sciences, Rishikesh-249203, India; ${ }^{3}$ Department of Biochemistry, All India Institute of Medical Sciences, Rishikesh-249203, India; ${ }^{4}$ Department of Medical Oncology, All India Institute of Medical Sciences, Rishikesh-249203; ${ }^{5}$ Department of Cancer Biology, Central Drug Research Institute, Lucknow-226031.

* To whom correspondence should be addressed:

Dr. Neeraj Jain

Department of Medical Oncology

All India Institute of Medical Sciences, Rishikesh-249203

Email: neeraj.monc@aiimsrishikesh.edu.in

Dr. Balram Ji Omar

Department of Microbiology,

All India Institute of Medical Sciences, Rishikesh-249203

Email: balram.micro@aiimsrishikesh.edu.in

Running Title: Pigment color in growth media determines antibiotic susceptibility of $P$. aeruginosa.

Keywords: Pigment, $\beta$-lactamase, MIC, Biofilm, Efflux

Abbreviations: AmpC: Ampicillin-hydrolyzing Cephalosporinase; ESBL: Extended Spectrum $\beta$ Lactamase; MBL: Metallo- $\beta$-Lactamase; EtBr-CW: Ethidium Bromide Cartwheel. 


\section{Graphical Abstract:}

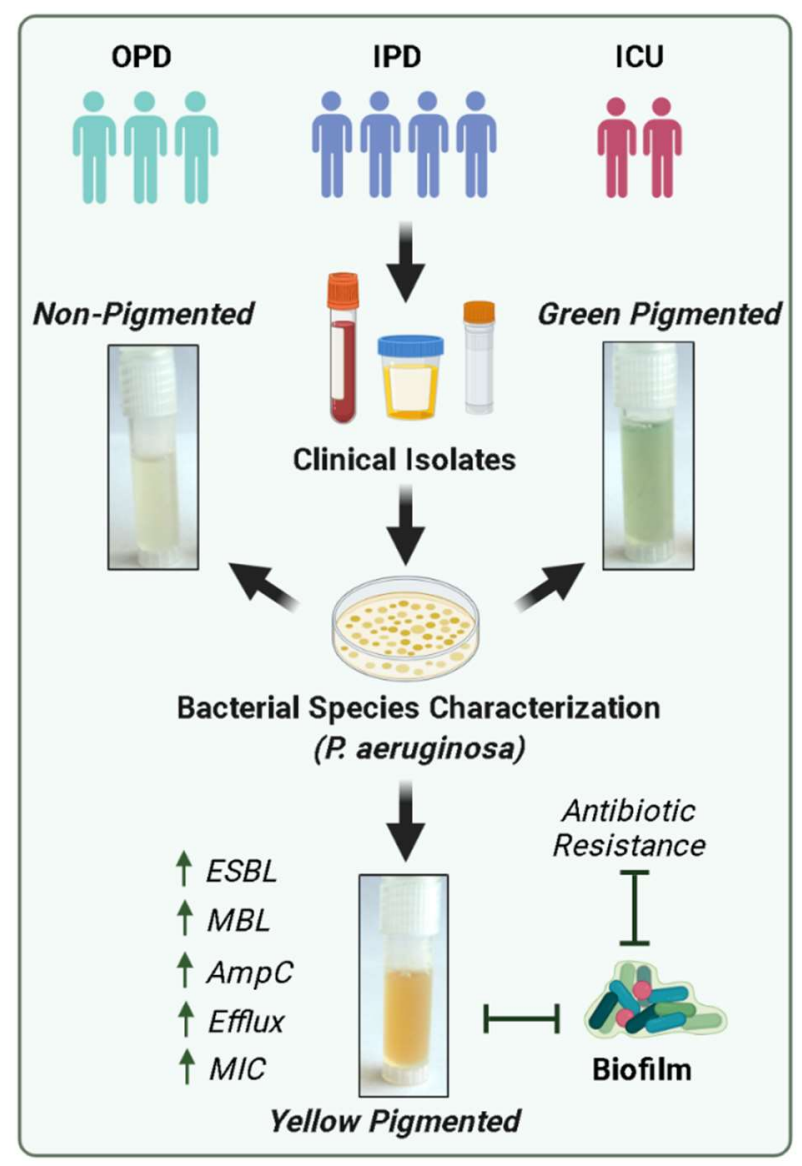

Pseudomonas aeruginosa is an adaptable bacterial pathogen that infects various organs, including the respiratory tract, vascular system, urinary tract, and central nervous system leading to high morbidity and mortality. Our primary focus of this study was to characterize $P$. aeruginosa clinical strains on the basis of pigment color production, determine its association to multidrug resistance behavior and ability to form biofilm. We identified yellow $(30.1 \%)$, green $(39.8 \%)$ and no pigment $(30.1 \%)$ producing strains from a total of 143 clinical isolates. Yellow pigment producing strains presented significant resistance to a class of antibiotics including $\beta$-lactam (91.5\%), aminoglycosides (70.5\%), and carbapenems (51.9\%) compared to green and non-pigmented strains. Importantly, $16.3 \%$ of yellow pigment producing strains was resistant to colistin where only $2.3 \%$ of non-pigmented and $1.8 \%$ of green pigmented strains were resistant to this agent. Moreover, yellow pigment producing strain were frequent producers of $\beta$-lactamase group of enzymes, ESBL (55.6\%), MBL (55.6\%), and AmpC $(50 \%)$ and displayed higher frequency of efflux positive group $(64.2 \%)$ compared to green $(7.14 \%)$ and non-pigmented one (28.5\%). Notably, green pigment producing strains when compared to non-pigmented groups also displayed antibiotic susceptibility behavior similar to yellow pigment producing strains. Although yellow pigment producing strains were strong biofilm producers, no significant association was identified between pigment and biofilm formation. Among pigmented and non-pigmented strains, majority of yellow pigment producing strains have shown MIC levels greater than the green and non-pigmented strains. Our study has demonstrated the impact of pigment coloration on susceptibility to antimicrobial agents where yellow pigment producing strains represent considerably a serious problem as due to lack of alternative agents against such transformed strain may collectively be associated with multidrug resistance development. 


\section{Introduction}

Difficult to treat multidrug-resistant (MDR) and extensively drug-resistant (XDR) Pseudomonas aeruginosa strains have become the leading cause of nosocomial infection to human beings and pose life challenging threat in health care settings to immunocompromised patients [1-4]. Though limiting-dose of antibiotics given in bits may strengthen sensitive bacteria and transforme them into resistant version, the other imperative factors such as bacterial genomic mutations and acquisition of resistance genes particularly those encoding extended-spectrum $\beta$-lactamases $(E S B L)$ or carbapenemases through horizontal gene transfer are also attributed to acquired resistance [5-8]. Notably, spread of so-called "high-risk" clones of $P$. aeruginosa poses a threat to global public health that requires extensive microbiological study and disease management to prevent their spread [8].

Pigments have functional roles in every organism, including lower organism such as microbes and higher ones, plants and animals. Microbial pigments can be primarily present in diffusible/water-soluble form where pigment is secreted out into the media and in non-diffusible form where pigment is retained within the organism. Most of the $P$. aeruginosa produces one or more extracellular pigments including pyoverdine (yellow-green and fluorescent), pyocyanin (blue-green), pyorubrin (red-brown), and pyomelanin (brown-black) [9,10]. These pigments are involved in multiple phenomenona such as quorum sensing network, virulence factor, antioxidant, and iron acquisition properties [11-13].

Functional implications of pigment production in $P$. aeruginosa pathogenesis have been acquainted with a number of studies. Laura et al have demonstrated contribution of pyomelanin production in $P$. aeruginosa provided resistance to oxidative stress and persistent chronic infection properties [13]. Notably, a strong correlation between pyoverdine production and virulence behavior of $P$. aeruginosa strain (isolated from cystic fibrosis patients) in a murine pneumonia model has been documented where disruption of pyoverdine production by specific inhibitor 5-fluorocytosine improved mice survival during infection and mitigated multidrug-resistant pathogenesis [14]. Pigment production appeared to be associated with virulent properties as expressions of virulence-associated genes (exoS, rhIA and rhIB) were more predominant in pigmented isolates than the non-pigmented clinical strains, suggesting that analyzing pigment production in clinical isolates can be a good initiative to detect virulence status of clinical strains [15]. 
Other important factors such as production of different class of enzymes called " $\beta$-lactamases" which include ESBLs, AmpC $\beta$-lactamases and metallo- $\beta$-lactamases (MBL) and non-enzymatic mechanism (overproduction of efflux pumps and outer membrane impermeability) are found to be associated with increased virulence and MDR behavior of $P$. aeruginosa isolated from clinical strains [16-19]. Notably, association of these factors with biofilm production which is another contributor of pathogenesis of $P$. aeruginosa has also been defined [20-23]. However, studies describing the clinical association between pigment production, MDR behavior and biofilm production are very limited [24,25]. In this study, we have presented association of pigment production status with MDR behavior, biofilm production, $\beta$-lactamases producing and efflux group of $P$. aeruginosa strains isolated from various clinical isolates at All India Institute of Medical Sciences, Rishikesh, India.

\subsection{Materials and methods}

\subsection{Ethics statement, and clinical samples}

This study was conducted after approval from Institutional Ethical Committee of All India Institute of Medical Sciences, Rishikesh, India. Protocol Number: ECR/736/Inst/UK/2015/RR-18. A total of 143 consecutive samples were selected of those identified to be infected with $P$. aeruginosa strain. Samples from various sites including pus, pleural fluid, urine, blood, sputum, and other discharges were collected from infected patients enrolled in respective departments, outside patients (OPD), inside patients (IPD) and intensive care unit (ICU). Samples after collection were immediately transported to the Microbiology lab and processed immediately as per routine hospital procedure of examination including bacterial identification, gram staining (HIMEDIA, K001), colony morphology (size, shape, texture, opacity), motility (hanging drop method), pigment production (identified by color production), oxidase reaction (Oxidase disc, DD018), and other routine microbiological procedures [26]. Further species identification for the isolates was carried out using Bruker's MALDI Biotyper Microbial Identification system (Bruker, USA).

\subsection{Pigment identification}

Color of pigment production was detected by qualitative observation of bacterial growth on preformed King's A medium agar (HiMedia, M1543). Inoculated bacteria were allowed to grow for 16 hours at $37^{\circ} \mathrm{C}$. Colonies those appeared blue-green in texture were considered pyocyanin 
producers, while-yellow colonies were considered as pyoverdine producers (Supplementary figure $1 \mathrm{~A})$.

\subsection{Antibiotic susceptibility test (AST)}

We have performed a disc diffusion method to test the AST of $P$. aeruginosa strains according to the Kirby-Bauer disk diffusion method on Muller-Hinton agar (MHA) [27]. We have also utilized an automated method for AST detection using a MicroScan WalkAway 96 Plus ID/AST system (Beckman Coulter, Inc., USA) equipped with software suitable for the interpretation of susceptibility testing results as per manufacturer's guidelines. Results of AST were interpreted and categorized as Susceptible (S), Intermediate (I) and Resistant (R) according to the Clinical Laboratory Standard Institute (CLSI) guidelines.

\subsection{Extended-spectrum $\beta$-lactamase detection.}

ESBL production from all $P$. aeruginosa isolates was detected by Beckman Coulter Microscan Walk-Away and double-disc synergy methods. ESBL production was screened by disc diffusion assay using ceftazidime (30 $\mathrm{gg})$ and ceftazidime/clavulanic acid $(30 / 10 \mu \mathrm{g})$ discs and Piperacillin $(100 \mu \mathrm{g})$ and Piperacillin/tazobactam $(100 / 10 \mu \mathrm{g})$ discs. The zones of inhibition for the ceftazidime and Piperacillin discs were compared to ceftazidime/clavulanic acid and Piperacillin/tazobactam discs. An increase in zone diameter in presence of tazobactam was confirmed as positive for ESBL production according to CLSI 2021 guideline. ESBL positive ATCC 27853 was used as a control strain. Bacteria showing resistance to at least three different classes of antibiotics were considered multidrug-resistant [28].

\subsection{Minimal inhibitory concentration (MIC) of MBL producing P. aeruginosa}

To detect MBL production by $P$. aeruginosa isolates, we performed RAPIDEC $₫$ CARBA NP test (Biomerieux-diagnostic) that is rapid and well adapted to detect carbapenemase producers as manufacturer instructions and as described previously [29]. A positive test for MBL producers corresponded to a color change from red to yellow or orange or light orange or dark orange. No color change indicated MBL-non producers strains (Supplementary Figure 2). MBL positive producers were further utilized for MIC determination for 7 antibiotics (Cefepime, Netilmicin, Gentamicin, Ciprofloxacin, Imipenem, Aztreonam, and Ceftazidime) respectively.

For MIC, we used antimicrobial gradient method Ezy MIC ${ }^{\text {TM }}$ Strips (Himedia). In brief, overnight culture of $P$. aeruginosa clinical isolates were diluted in peptone water to a turbidity of 0.5 
McFarland standards, followed by transfer of inoculum onto $\mathrm{MH}$ agar plate. We also utilized micro broth dilution method for MIC determination, where a 96-well micro-plate contained varying concentration of antibiotics. In brief, saline suspension of test strain equivalent to $0.5 \mathrm{McF}$ arland standards was prepared from a 72 hour-old subculture. A $100 \mu l$ volume of the suspension was added to each micro-plate with antibiotics incubated at 16 to 48 hours at $37^{\circ} \mathrm{C}$. We utilized ATCC 27853 as a reference strain. The MIC was defined as the lowest concentration of a test antibiotic that completely inhibited bacterial growth. MIC result was interpreted using CLSI guidelines (Supplementary Table 1).

\subsection{AmpC $\beta$-lactamases production phenotypic detection.}

AmpC $\beta$-lactamase production was phenotypically detected by the Ezy MICTM Strip (Himedia, EM081) according to the manufacturer's instructions. The test principle comprises a strip impregnated with a concentration gradient of cefotetan on one half of the strip and cefotetan with cloxacillin on the other half of the strip. MICs of cefotetan alone and cefotetan with cloxacillin were determined as recommended by the manufacturer. Ratios of cefotetan versus cefotetan/cloxacillin of $\geq 8$ were considered positive for AmpC beta-lactamase production.

\subsection{Efflux pumps detection by ethidium bromide cartwheel method.}

Trypticase Soy Agar (TSA) (HiMedia, M1969) plates containing ethidium bromide (EtBr) with varying concentrations ranging from 0 to $4 \mathrm{mg} / \mathrm{L}$ (these concentrations were determined according to the bacterial MICs for EtBr) were freshly prepared on the same day of experiment and kept protected from light. Overnight cultures of tested bacterial isolates were adjusted to a 0.5 McFarland turbidity standard. The TSA plates of 9.0-centimeter diameter was divided into ten to twelve sectors forming a cartwheel $(\mathrm{CW})$ pattern. The adjusted bacterial cultures were swabbed on the Et-Br-TSA plates starting from the plate's center to the margin. After incubating the plates at $37{ }^{\circ} \mathrm{C}$ for 16 hours, the plates were examined under a gel documentation system (Bio-rad, USA). The isolates were considered $\mathrm{Et}-\mathrm{Br}-\mathrm{CW}$-negative if they showed fluorescence emission at 0.5-1mg/L EtBr, EtBr-CW intermediate (emitting fluorescence at 2.0mg/L) or EtBrCW-positive (emitting fluorescence only at 3-4mg/L). Staphylococcus aureus ATCC 25923 strain was used as negative control for efflux experiment.

\subsection{Biofilm assay and quantification}

Biofilm formation assay was performed as described previously [30]. In brief, overnight culture of $P$. aeruginosa strain was diluted to OD of 0.5 according to McFarland standard. Suspension's 
culture was further diluted at 1:100 in 200 $\mu \mathrm{L}$ Luria Bertani broth (LB broth) (HiMedia M1245) and was then transferred into the sterile microtiter plate (96-well plates) and incubated in a static culture at $37^{\circ} \mathrm{C}$ for 3 days. The culture was discarded after three days and wells were washed

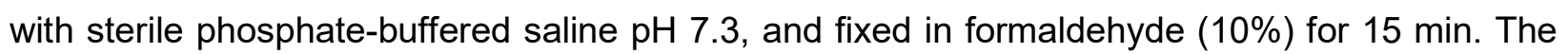
wells were air dried and stained with crystal violet to stain biofilm ( $0.1 \%$ in ethanol) (HiMedia) for 5 minutes. Wells were washed with deionized water to remove unbound dye and biofilm bound dye was eluted with ethanol. The optical density (OD) was measured at $550 \mathrm{~nm}$ using a plate reader (Eon Microplate Spectrophotometer by BioTek Instruments, Inc) and performed in triplicate. To categorise isolates as strong, moderate, and weak and no-biofilm producing strains, a cut off values of OD was set-up that represented mean \pm standard deviations (SD) of three independent experiments. The respective cut-off values of OD for strong $(1.0 \pm 0.2)$, moderate $(0.64 \pm 0.08)$, weak $(0.12 \pm 0.01)$, and no-biofilm $(0.03 \pm 0.00)$ were considered.

\subsection{Statistical analysis}

All data were analyzed and plotted using GraphPad Prism 5.02 software (GraphPad, La Jolla, CA, USA) and Microsoft excel. Differences between the groups and statistical significance were determined using Chi-square test. $P<0.05$ was considered statistically significant.

\subsection{Results}

\subsection{Prevalence of pigmented and non-pigmented $P$. aeruginosa clinical strains}

A total of 143 clinical isolates were obtained from OPD, IPD and ICU departments that were identified to be infected with $P$. aeruginosa. Among them, $92(64.5 \%)$ appeared in IPD department. Of the 143 patients, $93(65.1 \%)$ were males and $50(34.9 \%)$ were females $(P<0.05)$. Determination of mucoid and non-mucoid isolates was investigated phenotypically based on colony appearance and Congo red agar assay. Both non-mucoid 66 (46.1\%) and mucoid 77 (53.8\%) groups of $P$. aeruginosa were identified $(P>0.05)$ (Table 1$)$. Based on pigment production in Kings medium, $43(30.1 \%)$ of 143 archived isolates produced yellow pigment, whereas $57(39.8 \%)$ were green and $43(30.1 \%)$ with no pigment producing strains respectively $(P>0.05)$ (Figure 1A). As number of samples collected from IPD department was higher, therefore the relative distribution of green, yellow and no pigment producing strains were higher in IPD then the other hospital department (Figure 1B).

3.2 Antimicrobial resistance pattern of pigmented and non-pigmented $P$. aeruginosa clinical strains 
We next evaluated the antibiotic susceptibility pattern using a group of 14 anti-pseudomonal drugs. Pigmented strains (green and yellow producing strains) showed profound resistance to tested antibiotics compared to non-pigmented strains (Figure 1C). Among the pigmented and non-pigmented groups, the yellow pigment producing strains were more resistant to majorly of drugs tested then green and no pigment producing strains (Figure $1 \mathrm{C}$ and Supplementary Figure $1 \mathrm{~B}, \mathrm{C}$ ). The resistance frequency (in percentage) of non-pigmented, green, and yellow strains for represented antibiotics were: colistin $(2.3,1.8,16.3)$, ciprofloxacin $(23.3,24.6,53.5)$, doripenem (46.5, 49.1, 69.8), meropenem $(23.3,29.8,55.8)$, piperacillin/Tz (11.6, 19.3, 48.8), piperacillin $(27.9,15.8,37.2)$, ceftazidime $(34.9,54.4,74.4)$, and aztreonam $(53.5,40.4,53.5)$ respectively (Figure 1C). Similar results were obtained when patients were grouped into OPD, IPD, and ICU department, where yellow pigment producing strains presented a more frequent resistant pattern then green and non-pigmented strains (Figure 1D). Notably, patients from ICU showed profound resistance patterns in yellow pigment producing strains then IPD and OPD patients (Figure 1D and Table 2).

On distribution of antibiotics tested into different classes including aminoglycosides carbapenems, $\beta$-lactam and others (fluoroquinolones, polymyxin, sulfonamides), we found majority of $P$. aeruginosa strains were resistant to $\beta$-lactam class of antibiotics (Figure $2 A$ and Table 2). Based on pigment production, pigmented strains were more frequently resistant than the non-pigmented strains. We found yellow pigmented strains were strongly and significantly resistant to all classes of antibiotics followed by green and non-pigmented strains (Figure 2B-E). In $\beta$-lactam group, the prevalence of resistance in yellow strains $(91.5 \%)$ was highest followed by in green $(60.8 \%)$ and non-pigmented $(50.4 \%)$ strains. Similarly, in aminoglycosides class of antibiotics, the prevalence of resistance was $76.7 \%, 65.5 \%$ and $41.9 \%$; carbapenems class of antibiotics was $70.5 \%, 50.3 \%, 31.8 \%$, and for other class of antibiotics was $51.9 \%, 32.2 \%, 12.4 \%$ for yellow, green and non-pigmented strains respectively (Figure 2A).

3.3 ESBL, MBL, AmpC and efflux pump activity of pigmented and non-pigmented $P$. aeruginosa clinical strains

Our distribution analysis on the basis of ESBL, MBL and AmpC producing strains showed yellow pigmented $P$. aeruginosa were more frequent producers of ESBL (55.6\%), MBL (55.6\%), and AmpC (50\%) then green (ESBL, 30.5\%; MBL, 22.2\%; AmpC, 25\%) and non-pigmented (ESBL, 13.9\%; MBL, 22.2\%; AmpC, 25\%) strains (Figure 3A and Supplementary Figure 2). 
We next evaluated efflux activity of isolates by a fluorometric assay that detects EtBr efflux and presents the ability to pump EtBr out of the cell. We selected all $P$. aeruginosa clinical isolates that presented resistance towards ciprofloxacin (47 out of 143) by automated methods (Microscan Walk-Away 96 plus) and manual disc diffusion methods. Out of 47 ciprofloxacin resistant strains, 14 strains (29.8\%) did not retain $\mathrm{EtBr}$ at various levels when tested on agar plates containing $\mathrm{EtBr}$ (Supplementary Figure 3). Among 14 efflux positive strains, 9 of them (64.2\%) were yellow pigment producers, $1(7.14 \%)$ were green pigment producers and 4 (28.5\%) were non-pigmented strains (Figure $3 \mathrm{~A}$ ). Further characterization of efflux positive strain on the basis of high, moderate and low efflux activity, showed majority of yellow pigment producers had high efflux activity (Figure 3B).

We further evaluated the strains that were positive producers of ESBL, MBL, AmpC and showed efflux activity. We determine frequency of strains as single enzyme or efflux positive producers and co-producers strains (positive for two or more than two enzymes or enzyme plus efflux). The yellow and green pigment producing strains were equally distributed (46.6\%) among single enzyme/efflux positive producers, whereas non-pigmented strains were less frequent $(6.6 \%)$ (Figure $3 \mathrm{C}$ ). Where, yellow pigment producing strains were highly frequent $(61.9 \%)$, then green (19.04\%) and non-pigmented (19.04\%) strains among co-producers strains (Figure 3D).

\subsection{Biofilm production of pigmented and non-pigmented strains}

Mucoid and non-mucoid colonies are associated with biofilm production and resistance to antibiotics respectively. We do not observe significant difference in biofilm formation between mucoid and non-mucoid strains (Supplementary Figure 4A). However, mucoid strains were frequently resistant to piperacillin/Tz, piperacillin, meropenem, aztreonam and doripenem then non-mucoid strains (Supplementary Figure 4B). Among the mucoid and non-mucoid groups there was no relative difference observed in frequency of yellow, green and non-pigmented strains (Figure 4A). To test biofilm formation ability of clinical strains, we screened all 143 yellow, green and non-pigmented $P$. aeruginosa strains. We did not find correlation of antibiotic resistance pattern between biofilm and non-biofilm producers (Supplementary Figure 4C). Out of 143 strains, $106(74.1 \%)$ were biofilm producers, in which 34 (32.1\%) were yellow, 46 (43.4\%) green and 26 $(24.5 \%)$ non pigmented strains produced biofilm (Figure 4B). Based on OD received on microtiter plate, we have further categorized biofilm producers as strong, moderate and weak biofilm producers. Comparative analysis among yellow, green and non-pigmented strains showed 16 $(37.2 \%)$ of yellow, $17(29.8 \%)$ of green and 9 (20.9\%) of non-pigmented strains were strong biofilm 
producers. Whereas 6 (13.9\%) of yellow, 13 (22.8\%) of green and $12(27.9 \%)$ of non-pigmented were moderate biofilm producers; and $6(27.9 \%)$ of yellow, $13(28.1 \%)$ of green and $12(11.6 \%)$ of non-pigmented were weak biofilm producers (Figure 4C).

\subsection{MIC of MBL producing pigmented and non-pigmented strains.}

MBL producing $P$. aeruginosa strains are a threat to individuals and are associated with higher morbidity mortality rate especially in immunocompromised patients [31]. We have screened MIC level of all MBL producing clinical strains. Compared to reference strain ATCC 27853, all MBL positive clinical strains have shown MIC level greater than the MIC breakpoints recommended by CLSI guidelines with all 7 individual antibiotics tested (except the strain PA015 showed sensitivity to ciprofloxacin) (Supplementary Table 1). Yellow pigment producing strains have shown MIC levels even greater than green and non-pigmented strains (Figure 5A-I and Supplementary Table $1)$.

\subsection{Discussion}

The increasing incidence of MDR bacterial strains particularly for most common clinical pathogen $P$. aeruginosa in human isolates poses a significant challenge to identify new treatment strategies that can lead to high morbidity and mortality in hospitals. Fortunately, a number of rapid methods have been developed to identify such MDR species along with antibiotic susceptibility within a couple of hours from clinical isolates that have benefited to reduce increasing mortality [32-35]. Common characteristics for $P$. aeruginosa to represent the MDR behavior in clinical strains are presence of drug resistance genes or its mutant variety in bacterial plasmid or in its genome, high expression of $\beta$-lactamase group of enzymes, and up regulation of drug efflux pump have been documented [36-39]. Interestingly, a recent effort using proteomic profiling has characterized antibiotics sensitive and MDR clinical strains of $P$. aeruginosa that provided non-genetic changes associated with antibiotic susceptibility responses [40].

Pigment production such as pyocyanin and pyoverdin are important virulence factors that augment bacterial virulence via diverse mechanisms [13-15]. According to literature search and best of our knowledge, characterization of MDR signatures of $P$. aeruginosa from clinical strains on the basis of pigment production are very limited [13-15]. In this study, we have presented a strong association of pigment coloration produced by $P$. aeruginosa strains from clinical isolates with MDR behavior, efflux activity and biofilm formation. Primarily, we notified three major groups of $P$. aeruginosa: green, yellow and no pigment producing strains from our cohort of clinical 
samples. Note: we have also identified intermittent red and brown pigment producing strains, and we lacked their adequate numbers for analysis, therefore, we have excluded them from this study. Among the pigmented and non-pigmented strains, yellow pigment producing strain showed profound resistance behavior with majority of antibiotics tested in clinical samples archived from IPD and ICU department, suggesting that this yellow pigment producing strain can be a serious risk factor. Remarkable resistance pattern of yellow pigmented strain was noted for different classes of antibiotics groups including aminoglycosides, fluoroquinolones, carbapenem, sulfonamides, polymyxin and $\beta$-lactams.

Acquired resistance by the production of ESBL, MBL and AmpC enzymes is a common phenomenon $P$. aeruginosa [41]. Phenotypic methods applied in this study helped in detecting $P$. aeruginosa isolates producing various ESBL, MBL, AmpC, enzymes, and efflux activity against different antibiotics. Though, frequency of these enzymes producing strains from our cohort of 143 clinical isolates was not higher as shown by other studies, the prevalence of such producing organisms could depend on geographic origins, infection patterns, hospital infection control measures, and different departments within the same hospital [16,35]. Notably, our yellow pigment producing strains were more frequent enzyme producers and displayed co-occurrence with more than one enzyme/efflux positivity than green and non-pigmented strains. In addition, identification of MIC for MBL producing strains presented an augmented MIC level of yellow pigment producing strain than the CLSI MIC breakpoints.

Biofilm formation is an important mechanism $P$. aeruginosa survival causes considerable problems and these structures provoke greater resistance to the treatment with antibiotics. Inverse correlation between biofilm formation with expression of MDR genes has been identified in few studies [42,43]. In our study, though a significant number $(74.13 \%)$ of isolates formed biofilm, we did not observe a strong correlation of biofilm formation with pigment production.

Conclusively, green pigmented strains showed moderate resistance pattern when compared to yellow and non-pigmented strains suggesting that pigment producing strains could be associated to resistance to antimicrobial agents then the non-pigmented ones and requires comprehensive testing for antibiotics susceptibility pattern and to prevent detrimental effects before a treatment recommendation.

\subsection{Conclusion}


Our study has presented an association of pigment coloration with MDR behavior of $P$. aeruginosa isolated from various sources of clinical samples. Moreover, this study demonstrated ESBL, MBL, AmpC and Efflux mediated resistance among the different pigmented $P$. aeruginosa isolates. Though we have not identified the associated gene expression or genetic mutation or other mechanism connecting pigment production with MDR, which could be a further area of future research. We found yellow pigment color producing $P$. aeruginosa strains displayed resistant patterns to more than one type of antibiotic groups. Therefore, identification of right choice of antibiotic for treatment is critically important as misuse or overuse of antibiotics can cause significant risk of emergence of antibiotic resistance. Since pigment production is easy to determine, this might be a good starting point to identify the multi-drug resistance status of an isolate. However, further study is required to confirm this observation.

Author Contributions: A.K. designed \& performed all the experiments, generated tables \& figures. NJ contributed to write the manuscript. R.Y., P.R., V.S., K.K., NJ and B.J.O, generated laboratory resources, had proofread and approved the final manuscript. All authors have read and agreed to the published version of the manuscript.

Funding: This work is supported by an intramural fund from All India Institute Medical Sciences, Rishikesh provided to A.K. as Ph.D. fellowship (319/IEC/Ph.D./2018).

Conflicts of interest: The authors declare that they have no competing interests.

\section{References:}

1. Abdelrahman, D.N.; Taha, A.A.; Dafaallah, M.M.; Mohammed, A.A.; El Hussein, A.R.M.; Hashim, A.I.; Hamedelnil, Y.F.; Altayb, H.N. beta-lactamases (bla TEM, bla SHV, bla CTXM-1, bla VEB, bla OXA-1 ) and class $C$ beta-lactamases gene frequency in Pseudomonas aeruginosa isolated from various clinical specimens in Khartoum State, Sudan: a cross sectional study. F1000Res 2020, 9, 774, doi:10.12688/f1000research.24818.3.

2. Gale, M.J.; Maritato, M.S.; Chen, Y.L.; Abdulateef, S.S.; Ruiz, J.E. Pseudomonas aeruginosa causing inflammatory mass of the nasopharynx in an immunocompromised HIV infected patient: A mimic of malignancy. IDCases 2015, 2, 40-43, doi:10.1016/j.idcr.2015.01.004.

3. Gomila, A.; Carratala, J.; Badia, J.M.; Camprubi, D.; Piriz, M.; Shaw, E.; Diaz-Brito, V.; Espejo, E.; Nicolas, C.; Brugues, M., et al. Preoperative oral antibiotic prophylaxis reduces Pseudomonas aeruginosa surgical site infections after elective colorectal surgery: a multicenter prospective cohort study. BMC Infect Dis 2018, 18, 507, doi:10.1186/s12879-018-3413-1.

4. Pang, Z.; Raudonis, R.; Glick, B.R.; Lin, T.J.; Cheng, Z. Antibiotic resistance in Pseudomonas aeruginosa: mechanisms and alternative therapeutic strategies. Biotechnol Adv 2019, 37, 177-192, doi:10.1016/j.biotechadv.2018.11.013.

5. Gjini, E.; Pauperio, F.F.S.; Ganusov, V.V. Treatment timing shifts the benefits of short and long antibiotic treatment over infection. Evol Med Public Health 2020, 2020, 249-263, doi:10.1093/emph/eoaa033.

6. Poole, K. Pseudomonas aeruginosa: resistance to the max. Front Microbiol 2011, 2, 65, doi:10.3389/fmicb.2011.00065. 
7. Breidenstein, E.B.; de la Fuente-Nunez, C.; Hancock, R.E. Pseudomonas aeruginosa: all roads lead to resistance. Trends Microbiol 2011, 19, 419-426, doi:10.1016/j.tim.2011.04.005.

8. Oliver, A.; Mulet, X.; Lopez-Causape, C.; Juan, C. The increasing threat of Pseudomonas aeruginosa high-risk clones. Drug Resist Updat 2015, 21-22, 41-59, doi:10.1016/j.drup.2015.08.002.

9. Krieg, N.R. Bergey's Manual of Systematic Bacteriology. 2nd ed.; Springer-Verlag: 2010.

10. Prince, A.S. Principles and Practice of Pediatric Infectious Diseases. Forth ed.; 2012.

11. Dietrich, L.E.; Price-Whelan, A.; Petersen, A.; Whiteley, M.; Newman, D.K. The phenazine pyocyanin is a terminal signalling factor in the quorum sensing network of Pseudomonas aeruginosa. Mol Microbiol 2006, 61, 1308-1321, doi:10.1111/j.1365-2958.2006.05306.x.

12. Elbargisy, R.M. Optimization of nutritional and environmental conditions for pyocyanin production by urine isolates of Pseudomonas aeruginosa. Saudi J Biol Sci 2021, 28, 993-1000, doi:10.1016/j.sjbs.2020.11.031.

13. Ketelboeter, L.M.; Potharla, V.Y.; Bardy, S.L. NTBC treatment of the pyomelanogenic Pseudomonas aeruginosa clinical isolate PA1111 inhibits pigment production and increases sensitivity to oxidative stress. Curr Microbiol 2014, 69, 343-348, doi:10.1007/s00284-014-0593-9.

14. Kang, D.; Revtovich, A.V.; Chen, Q.; Shah, K.N.; Cannon, C.L.; Kirienko, N.V. PyoverdineDependent Virulence of Pseudomonas aeruginosa Isolates From Cystic Fibrosis Patients. Front Microbiol 2019, 10, 2048, doi:10.3389/fmicb.2019.02048.

15. Finlayson, E.A.; Brown, P.D. Comparison of antibiotic resistance and virulence factors in pigmented and non-pigmented Pseudomonas aeruginosa. West Indian Med J 2011, 60, 24-32.

16. Peymani, A.; Naserpour-Farivar, T.; Zare, E.; Azarhoosh, K.H. Distribution of blaTEM, blaSHV, and blaCTX-M genes among ESBL-producing P. aeruginosa isolated from Qazvin and Tehran hospitals, Iran. J Prev Med Hyg 2017, 58, E155-E160.

17. Rawat, D.; Nair, D. Extended-spectrum beta-lactamases in Gram Negative Bacteria. J Glob Infect Dis 2010, 2, 263-274, doi:10.4103/0974-777X.68531.

18. Oberoi, L.; Singh, N.; Sharma, P.; Aggarwal, A. ESBL, MBL and Ampc beta Lactamases Producing Superbugs - Havoc in the Intensive Care Units of Punjab India. J Clin Diagn Res 2013, 7, 70-73, doi:10.7860/JCDR/2012/5016.2673.

19. Cunrath, O.; Meinel, D.M.; Maturana, P.; Fanous, J.; Buyck, J.M.; Saint Auguste, P.; Seth-Smith, H.M.B.; Korner, J.; Dehio, C.; Trebosc, V., et al. Quantitative contribution of efflux to multi-drug resistance of clinical Escherichia coli and Pseudomonas aeruginosa strains. EBioMedicine 2019, 41, 479-487, doi:10.1016/j.ebiom.2019.02.061.

20. Moradali, M.F.; Ghods, S.; Rehm, B.H. Pseudomonas aeruginosa Lifestyle: A Paradigm for Adaptation, Survival, and Persistence. Front Cell Infect Microbiol 2017, 7, 39, doi:10.3389/fcimb.2017.00039.

21. Heydari, S.; Eftekhar, F. Biofilm Formation and beta-Lactamase Production in Burn Isolates of Pseudomonas aeruginosa. Jundishapur J Microbio/ 2015, 8, e15514, doi:10.5812/jjm.15514.

22. Asadpour, L. Antimicrobial resistance, biofilm-forming ability and virulence potential of Pseudomonas aeruginosa isolated from burn patients in northern Iran. J Glob Antimicrob Resist 2018, 13, 214-220, doi:10.1016/j.jgar.2018.01.018.

23. Szemeredi, N.; Kincses, A.; Rehorova, K.; Hoang, L.; Salardon-Jimenez, N.; Sevilla-Hernandez, C.; Viktorova, J.; Dominguez-Alvarez, E.; Spengler, G. Ketone- and Cyano-Selenoesters to Overcome Efflux Pump, Quorum-Sensing, and Biofilm-Mediated Resistance. Antibiotics (Basel) 2020, 9, doi:10.3390/antibiotics9120896.

24. Milojkovic, M.; Nenadovic, Z.; Stankovic, S.; Bozic, D.D.; Nedeljkovic, N.S.; Cirkovic, I.; Petrovic, M.; Dimkic, I. Phenotypic and genetic properties of susceptible and multidrug-resistant Pseudomonas aeruginosa isolates in Southern Serbia. Arh Hig Rada Toksikol 2020, 71, 231-250, doi:10.2478/aiht-2020-71-3418.

25. Meirelles, L.A.; Newman, D.K. Both toxic and beneficial effects of pyocyanin contribute to the lifecycle of Pseudomonas aeruginosa. Mol Microbiol 2018, 110, 995-1010, doi:10.1111/mmi.14132.

26. Collee JG, D.J., Fraser AG, Marmion BP, Simmons A. Laboratory strategy in the diagnosis of infective syndromes. Mackie and McCartney practical medical microbiology: 1996; Vol. 14, pp. 5394.

27. Hudzicki, J. Kirby-Bauer Disk Diffusion Susceptibility Test ASM: 2009. 
28. Magiorakos, A.P.; Srinivasan, A.; Carey, R.B.; Carmeli, Y.; Falagas, M.E.; Giske, C.G.; Harbarth, S.; Hindler, J.F.; Kahlmeter, G.; Olsson-Liljequist, B., et al. Multidrug-resistant, extensively drugresistant and pandrug-resistant bacteria: an international expert proposal for interim standard definitions for acquired resistance. Clin Microbiol Infect 2012, 18, 268-281, doi:10.1111/j.14690691.2011.03570.x.

29. Poirel, L.; Nordmann, P. Rapidec Carba NP Test for Rapid Detection of Carbapenemase Producers. J Clin Microbiol 2015, 53, 3003-3008, doi:10.1128/JCM.00977-15.

30. Tang, J.; Wang, W.; Chu, W. Antimicrobial and Anti-Quorum Sensing Activities of Phlorotannins From Seaweed (Hizikia fusiforme). Front Cell Infect Microbiol 2020, 10, 586750, doi:10.3389/fcimb.2020.586750.

31. Hong, D.J.; Bae, I.K.; Jang, I.H.; Jeong, S.H.; Kang, H.K.; Lee, K. Epidemiology and Characteristics of Metallo-beta-Lactamase-Producing Pseudomonas aeruginosa. Infect Chemother 2015, 47, 81 97, doi:10.3947/ic.2015.47.2.81.

32. Jackson, N.; Borges, C.A.; Tarlton, N.J.; Resendez, A.; Milton, A.K.; de Boer, T.R.; Butcher, C.R.; Murthy, N.; Riley, L.W. A rapid, antibiotic susceptibility test for multidrug-resistant, Gram-negative bacterial uropathogens using the biochemical assay, DETECT. J Microbiol Methods 2021, 182, 106160, doi:10.1016/j.mimet.2021.106160.

33. Demord, A.; Poirel, L.; D'Emidio, F.; Pomponio, S.; Nordmann, P. Rapid ESBL NP Test for Rapid Detection of Expanded-Spectrum beta-Lactamase Producers in Enterobacterales. Microb Drug Resist 2020, 10.1089/mdr.2020.0391, doi:10.1089/mdr.2020.0391.

34. Rudresh, S.M.; Ravi, G.S.; Sunitha, L.; Hajira, S.N.; Kalaiarasan, E.; Harish, B.N. Simple, rapid, and cost-effective modified Carba NP test for carbapenemase detection among Gram-negative bacteria. J Lab Physicians 2017, 9, 303-307, doi:10.4103/JLP.JLP_138_16.

35. Begum, S.; Salam, M.A.; Alam Kh, F.; Begum, N.; Hassan, P.; Haq, J.A. Detection of extended spectrum beta-lactamase in Pseudomonas spp. isolated from two tertiary care hospitals in Bangladesh. BMC Res Notes 2013, 6, 7, doi:10.1186/1756-0500-6-7.

36. El Shamy, A.A.; Zakaria, Z.; Tolba, M.M.; Salah Eldin, N.; Rabea, A.T.; Tawfick, M.M.; Nasser, H.A. AmpC beta-Lactamase Variable Expression in Common Multidrug-Resistant Nosocomial Bacterial Pathogens from a Tertiary Hospital in Cairo, Egypt. Int J Microbiol 2021, 2021, 6633888, doi:10.1155/2021/6633888.

37. Hosu, M.C.; Vasaikar, S.D.; Okuthe, G.E.; Apalata, T. Detection of extended spectrum betalactamase genes in Pseudomonas aeruginosa isolated from patients in rural Eastern Cape Province, South Africa. Sci Rep 2021, 11, 7110, doi:10.1038/s41598-021-86570-y.

38. Tooke, C.L.; Hinchliffe, P.; Bragginton, E.C.; Colenso, C.K.; Hirvonen, V.H.A.; Takebayashi, Y.; Spencer, J. beta-Lactamases and beta-Lactamase Inhibitors in the 21st Century. J Mol Biol 2019, 431, 3472-3500, doi:10.1016/j.jmb.2019.04.002.

39. Manchanda, V.; Singh, N.P. Occurrence and detection of AmpC beta-lactamases among Gramnegative clinical isolates using a modified three-dimensional test at Guru Tegh Bahadur Hospital, Delhi, India. J Antimicrob Chemother 2003, 51, 415-418, doi:10.1093/jac/dkg098.

40. Liew, S.M.; Puthucheary, S.D.; Rajasekaram, G.; Chai, H.C.; Chua, K.H. Proteomic profiling of clinical and environmental strains of Pseudomonas aeruginosa. Mol Biol Rep 2021, 48, 2325-2333, doi:10.1007/s11033-021-06262-8.

41. Paterson, D.L.; Bonomo, R.A. Extended-spectrum beta-lactamases: a clinical update. Clin Microbiol Rev 2005, 18, 657-686, doi:10.1128/CMR.18.4.657-686.2005.

42. Yamani, L.; Alamri, A.; Alsultan, A.; Alfifi, S.; Ansari, M.A.; Alnimr, A. Inverse correlation between biofilm production efficiency and antimicrobial resistance in clinical isolates of Pseudomonas aeruginosa. Microb Pathog 2021, 10.1016/j.micpath.2021.104989, 104989, doi:10.1016/j.micpath.2021.104989.

43. Kamali, E.; Jamali, A.; Ardebili, A.; Ezadi, F.; Mohebbi, A. Evaluation of antimicrobial resistance, biofilm forming potential, and the presence of biofilm-related genes among clinical isolates of Pseudomonas aeruginosa. BMC Res Notes 2020, 13, 27, doi:10.1186/s13104-020-4890-z. 
Table 1: Demographic and Clinical Characteristics of $P$. aeruginosa infected patients from OPD, IPD, and ICU.

\begin{tabular}{|c|c|c|c|c|c|}
\hline \multicolumn{2}{|l|}{ Characteristics } & $\begin{array}{c}\text { OPD n }\{\%\} \\
36\{25.1\}\end{array}$ & $\begin{array}{l}\text { IPD n }\{\%\} \\
n=92\{64.5\}\end{array}$ & $\begin{array}{l}\text { ICU n }\{\%\} \\
n=15\{10.4\}\end{array}$ & $\begin{array}{c}\text { Total n }\{\%\} \\
n=143\end{array}$ \\
\hline Age & & $41 \pm 16.3$ & $40.5 \pm 17.3$ & $47.5 \pm 18.5$ & \\
\hline \multirow[t]{2}{*}{ Gender } & Male & $23\{63.8\}$ & $58\{63\}$ & $12\{80\}$ & $93\{65.1\}$ \\
\hline & Female & $13\{36.1\}$ & $34\{36.9\}$ & $3\{20\}$ & $50\{34.9\}$ \\
\hline \multirow[t]{3}{*}{ Pigment Status } & No Pigment & $14\{38.8\}$ & $26\{28.2\}$ & $3\{20\}$ & $43\{30\}$ \\
\hline & Green & $15\{26.3\}$ & $36\{39.1\}$ & $6\{40\}$ & $57\{39.8\}$ \\
\hline & Yellow & $7\{19.4\}$ & $30\{32.6\}$ & $6\{40\}$ & $43\{30\}$ \\
\hline \multirow[t]{2}{*}{ Mucus status } & Non-mucoid & $20\{55.5\}$ & $38\{41.3\}$ & $7\{46.6\}$ & $66\{46.1\}$ \\
\hline & Mucoid & $16\{44.4\}$ & $54\{58.6\}$ & $8\{53.3\}$ & $77\{53.8\}$ \\
\hline
\end{tabular}

Table 2: Resistance pattern of $P$. aeruginosa strains represented in percentage isolated from patients visited to OPD, IPD, and ICU for listed classes of antibiotics.

\begin{tabular}{|c|c|c|c|c|c|c|c|c|c|c|c|}
\hline \multirow{2}{*}{$\begin{array}{l}\text { Groups of } \\
\text { Antibiotics }\end{array}$} & \multirow[t]{2}{*}{ Antibiotics } & \multicolumn{3}{|c|}{ Non-pigment } & \multicolumn{3}{|l|}{ Green } & \multicolumn{3}{|l|}{ yellow } & \multirow{2}{*}{\begin{tabular}{|l|} 
Total \\
143 \\
\end{tabular}} \\
\hline & & $\begin{array}{l}\text { OPD } \\
\{n=14\}\end{array}$ & $\begin{array}{l}\text { IPD } \\
\{n=26\}\end{array}$ & $\begin{array}{l}\text { ICU } \\
\{n=3\}\end{array}$ & $\begin{array}{l}\text { OPD } \\
\{n=15\}\end{array}$ & $\begin{array}{l}\text { IPD } \\
\{n=36\}\end{array}$ & $\begin{array}{l}\text { ICU } \\
\{n=6\}\end{array}$ & $\begin{array}{l}\text { OPD } \\
\{n=7\}\end{array}$ & $\begin{array}{l}\text { IPD } \\
\{n=30\}\end{array}$ & $\begin{array}{l}\text { ICU } \\
\{n=6\}\end{array}$ & \\
\hline \multirow[t]{3}{*}{ Aminoglycosides } & Tobramycin & $3\{21.4\}$ & $12\{46.1\}$ & $1\{33.3\}$ & $10\{66.6\}$ & $20\{55.5\}$ & $5\{83.3\}$ & $3\{42.8\}$ & $18\{60\}$ & $5\{83.3\}$ & $77\{53.8\}$ \\
\hline & Gentamicin & $3\{21.4\}$ & $16\{61.5\}$ & $0\{0\}$ & $10\{66.6\}$ & $25\{69.4\}$ & $5\{83.3\}$ & $6\{85.7\}$ & $25\{83.3\}$ & $6\{100\}$ & $96\{67.1\}$ \\
\hline & Amikacin & $4\{28.5\}$ & $15\{57.6\}$ & $0\{0\}$ & $9\{60\}$ & $23\{63.8\}$ & $5\{83.3\}$ & $5\{71.4\}$ & $25\{83.3\}$ & $6\{100\}$ & $92\{64.3\}$ \\
\hline \multirow[t]{5}{*}{ B-Lactams } & Aztreonam & $5\{35.7\}$ & $15\{57.6\}$ & $3\{100\}$ & $7\{46.6\}$ & $16\{44.4\}$ & $0\{0\}$ & $2\{28.5\}$ & $19\{63.3\}$ & $2\{33.3\}$ & $69\{48.2\}$ \\
\hline & Ceftazidime & $2\{14.2\}$ & $13\{50\}$ & $0\{0\}$ & $8\{53.3\}$ & $19\{52.7\}$ & $4\{66.6\}$ & $6\{85.7\}$ & $22\{73.3\}$ & $4\{66.6\}$ & $78\{54.5\}$ \\
\hline & Cefepime & $1\{7.1\}$ & $9\{34.6\}$ & $0\{0\}$ & $7\{46.6\}$ & $18\{50\}$ & $5\{83.3\}$ & $3\{42.8\}$ & $19\{63.3\}$ & $4\{66.6\}$ & $66\{46.1\}$ \\
\hline & Piperacillin & $3\{21.4\}$ & $9\{34.6\}$ & $0\{0\}$ & $2\{13.3\}$ & $7\{19.4\}$ & $0\{0\}$ & $3\{42.8\}$ & $19\{63.3\}$ & $3\{50\}$ & $46\{32.1\}$ \\
\hline & Piperacillin/Tz & $0\{0\}$ & $5\{19.2\}$ & $0\{0\}$ & $4\{26.6\}$ & $6\{16.6\}$ & $1\{16.6\}$ & $3\{42.8\}$ & $15\{50\}$ & $3\{33.3\}$ & $36\{25.1\}$ \\
\hline \multirow[t]{3}{*}{ Carbapenem } & Imipenem & $1\{7.1\}$ & $10\{38.4\}$ & $0\{0\}$ & $12\{80\}$ & $25\{69.4\}$ & $4\{66.6\}$ & $6\{85.7\}$ & $25\{83.3\}$ & $6\{100\}$ & $89\{62.2\}$ \\
\hline & Meropenem & $1\{7.1\}$ & $9\{34.6\}$ & $0\{0\}$ & $5\{33.3\}$ & $10\{27.7\}$ & $2\{33.3\}$ & $2\{28.5\}$ & $18\{60\}$ & $4\{66.6\}$ & $51\{35.6\}$ \\
\hline & Doripenem & $5\{35.7\}$ & $15\{57.6\}$ & $0\{0\}$ & $8\{53.3\}$ & $16\{44.4\}$ & $4\{66.6\}$ & $4\{57.1\}$ & $21\{70\}$ & $5\{83.3\}$ & $78\{45.5\}$ \\
\hline Fluoroquinolones & Ciprofloxacin & $2\{14.2\}$ & $8\{30.7\}$ & $0\{0\}$ & $5\{33.3\}$ & $6\{16.6\}$ & $3\{50\}$ & $3\{42.8\}$ & $18\{60\}$ & $2\{33.3\}$ & $47\{32.8\}$ \\
\hline Polymyxin & Colistin & $0\{0\}$ & $1\{3.8\}$ & $0\{0\}$ & $0\{0\}$ & $1\{2.7\}$ & $0\{0\}$ & $1\{14.2\}$ & $5\{16.6\}$ & $1\{16.6\}$ & $9\{6.2\}$ \\
\hline Sulfonamides & Co-trimoxazole & $0\{0\}$ & $5\{19.2\}$ & $0\{0\}$ & $11\{73.3\}\}$ & $24\{66.6\}$ & $5\{83.3\}$ & $4\{57.1\}$ & $27\{90\}$ & $6\{100\}$ & $82\{57.3\}$ \\
\hline
\end{tabular}


A

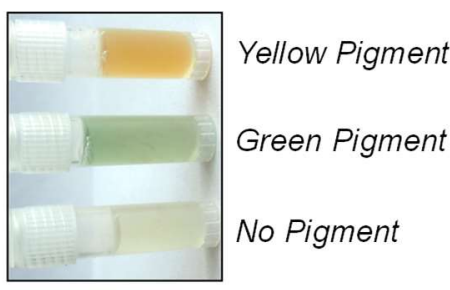

B

\begin{tabular}{|c|c|c|c|c|}
\hline & $O P D$ & $I P D$ & \multicolumn{2}{|c|}{ ICU } \\
\hline No Pigment & 14[ & & 26 & 3 \\
\hline Green & 15 & & 36 & 6 \\
\hline Yellow & 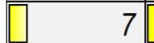 & & 30[ & 6 \\
\hline
\end{tabular}

pigmented (green and yellow) and non-pigmented clinical strain.
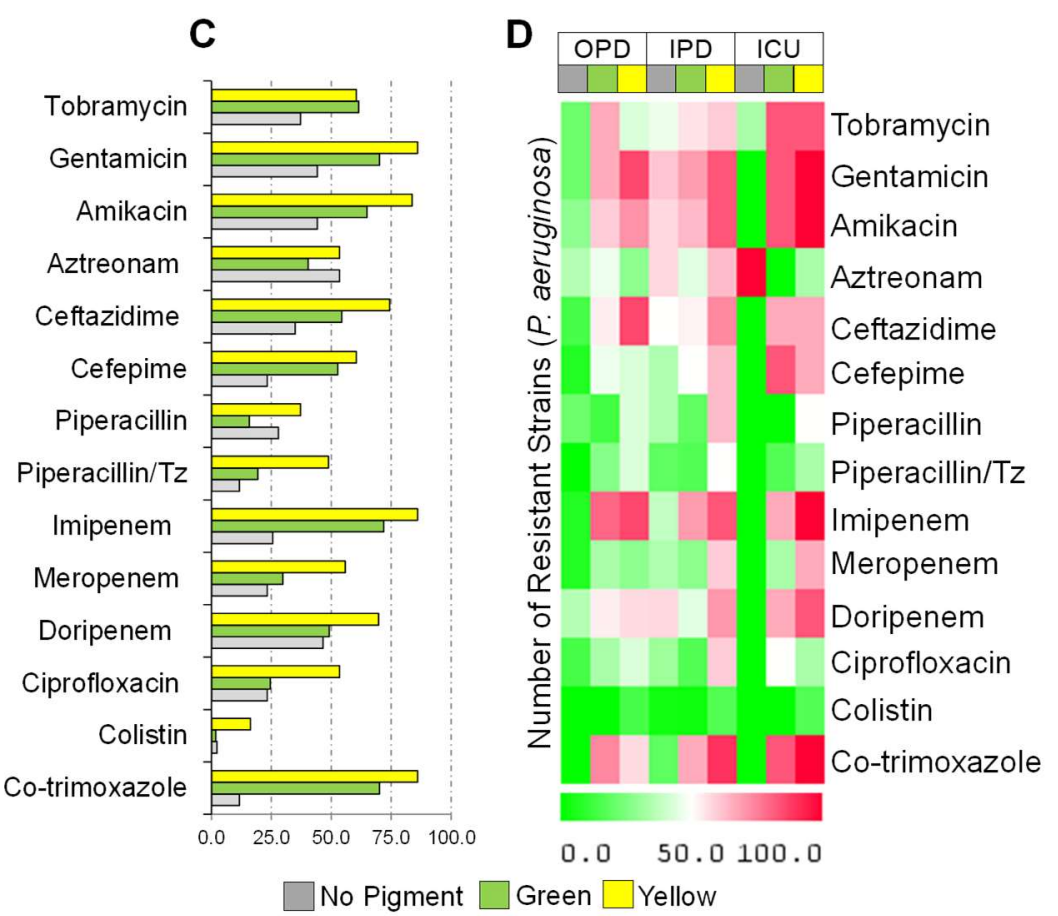

Figures 1: Antibiotic resistance pattern of clinical $\boldsymbol{P}$. aeruginosa strains: $(A)$ Represented image of pigmented and non-pigmented strains from different departments. (C) Frequency of green, yellow and no pigment producing strains with respect to represented antibiotics. (D) Heat-map representing number of respective antibiotic resistant strains relative to pigmented and non-pigmented group in OPD, IPD and ICU department. 
A

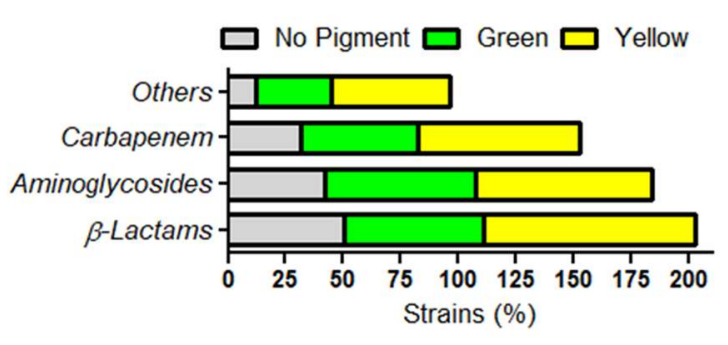

C

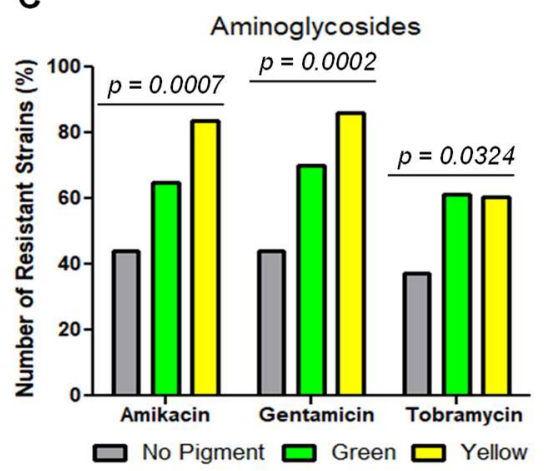

D
B
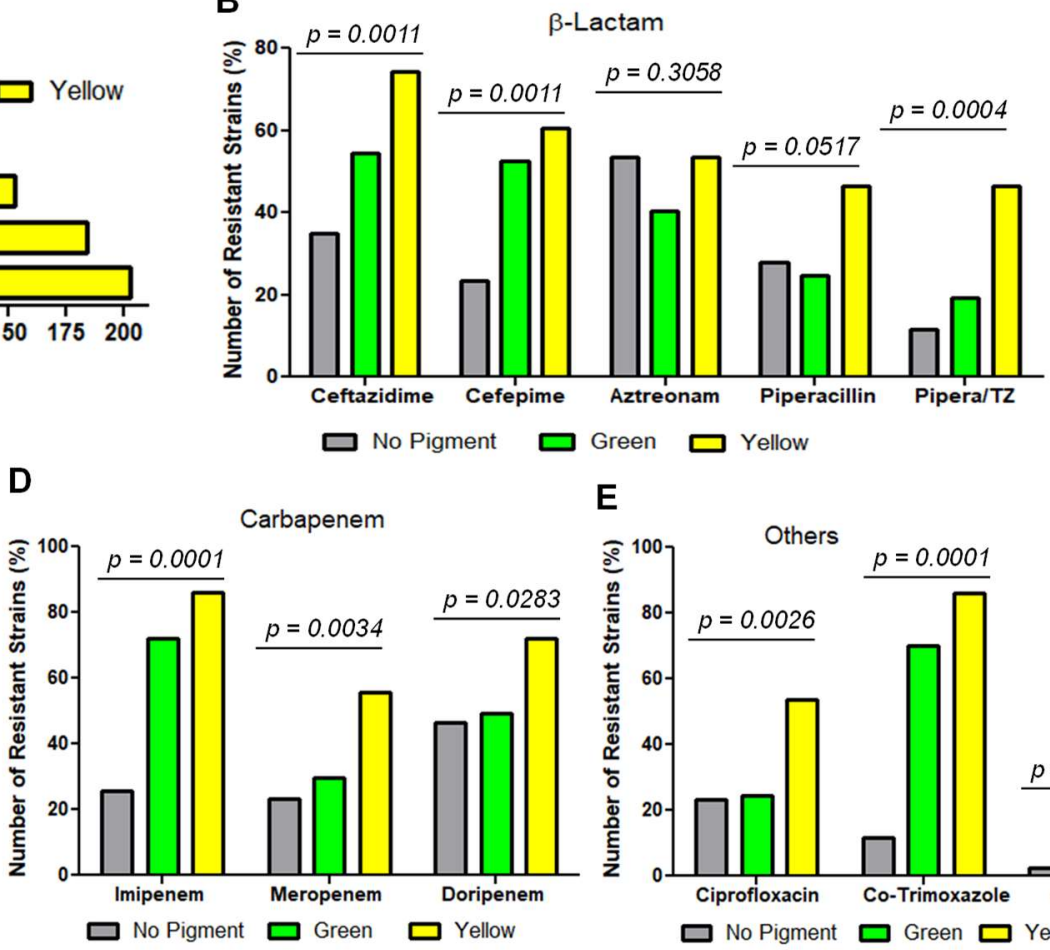

E

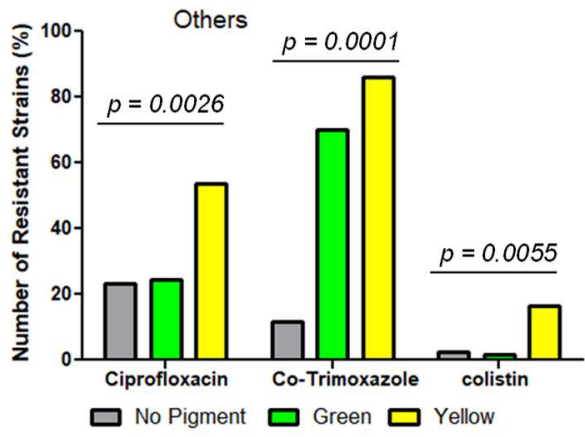

Figures 2: Antibiotic susceptibility pattern according to different classes: (A) P. aeruginosa clinical strains representing antibiotic susceptibility to different class of antibiotics. Resistance pattern of green, yellow and no pigment producing strains among antimicrobial groups of $(B)$ aminoglycosides, $(C) \beta$-Lactam, (D) carbapenem, (E) others including fluoroquinolones (ciprofloxacin), sulfonamides (co-trimoxazole), polymyxin (colistin). $P<0.05$ is considered statistically significant (Chi Square test). 

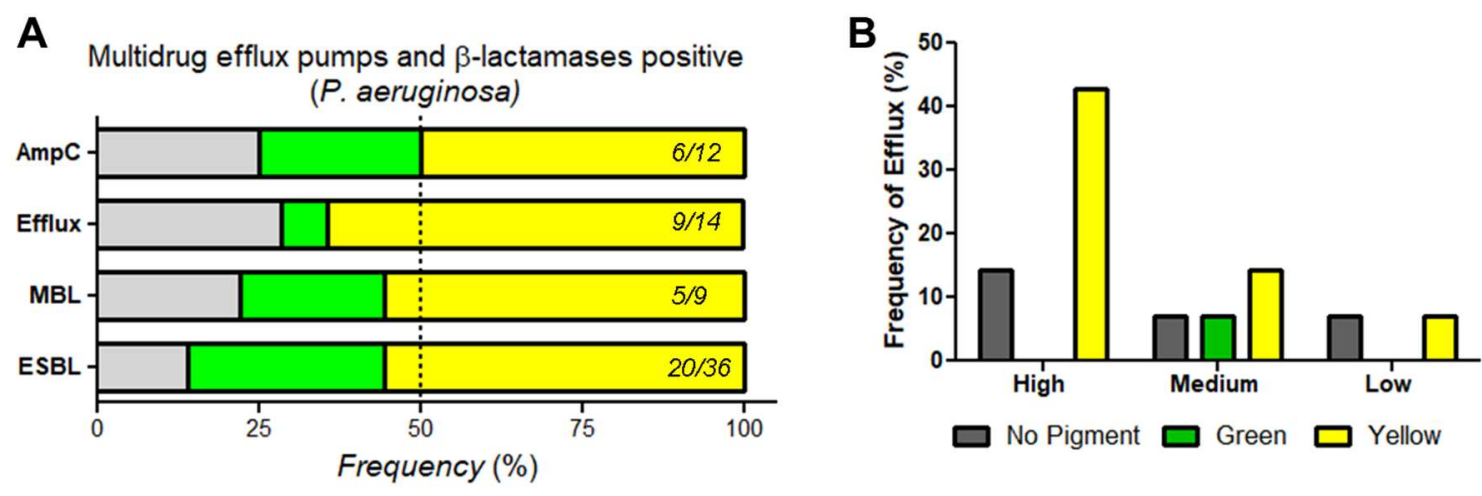

Single enzyme or efflux
positive group $(\%)$

D Combinatorial enzyme or efflux
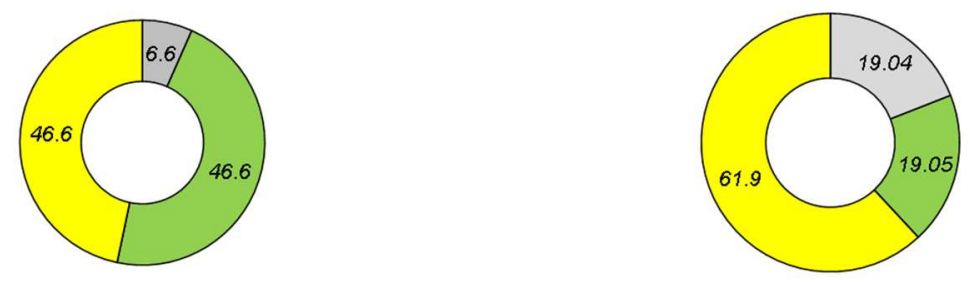

口No Pigment aGreen aYellow

口No Pigment aGreen aYellow

Figures 3: Distribution of antibiotic susceptibility pattern according to efflux and $\beta$-lactamases enzyme producing pigmented and non-pigmented $\boldsymbol{P}$. aeruginosa clinical strains: (A) Frequency of Extended-Spectrum, matello $\beta$-Lactamase, AmpC- $\beta$-Lactamase and efflux positive strains in pigmented (green and yellow) and non-pigmented groups. (B) Frequency of high, moderate and low efflux producing green, yellow and non-pigmented strains. Number of yellow, green and non-pigmented strains shown in percentage comprises single $(C)$ or combinatorial $\beta$-lactamases producing strains. 


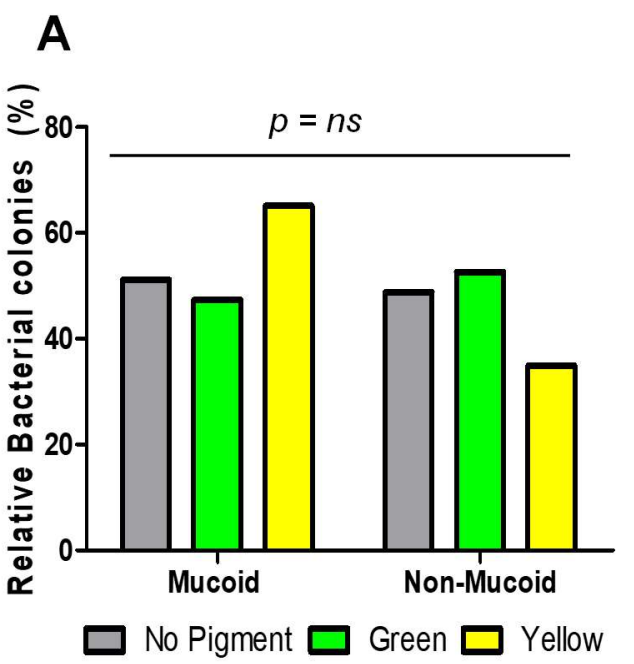

\section{C}

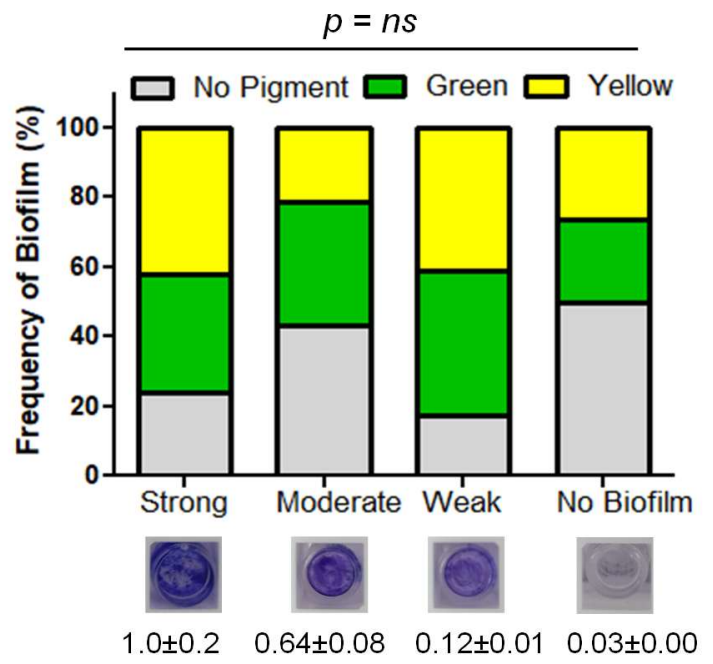

\section{B Biofilm producers}

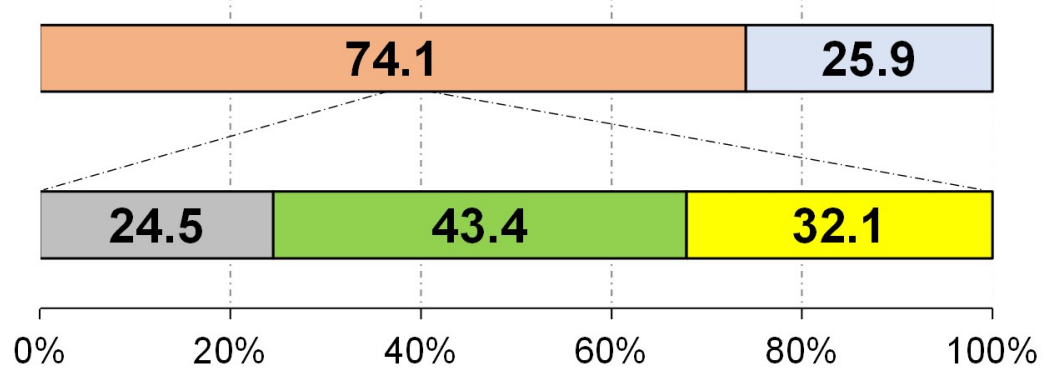

Figure 4: Biofilm producing property of pigmented and non-pigmented $\boldsymbol{P}$. aeruginosa strains: (A) Relative percentage of pigmented and non-pigmented strains confined mucoid or non-mucoid phenotype. (B) Percentage of biofilm producing and no biofilm producing pigmented and non-pigmented strains. (C) Frequency of strong, moderate, and weak biofilm producers among no pigmented and pigmented strains, $n s=$ not significant (Chi Square test). 
A

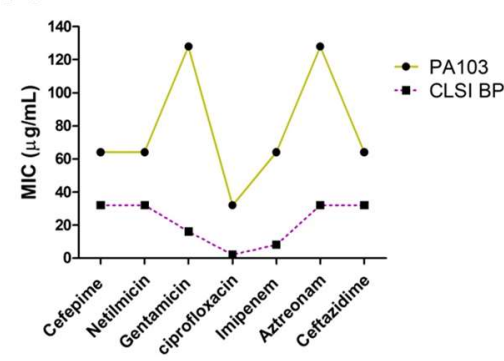

D

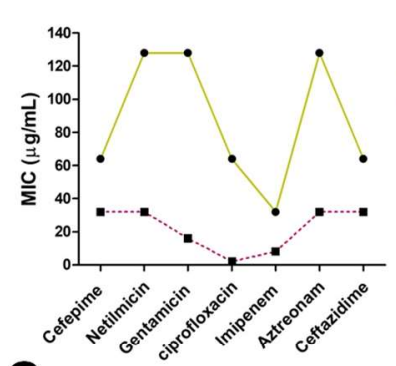

G

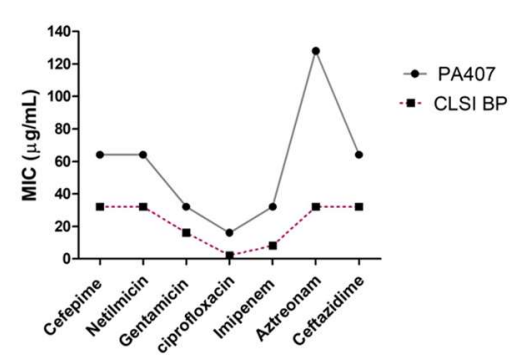

B

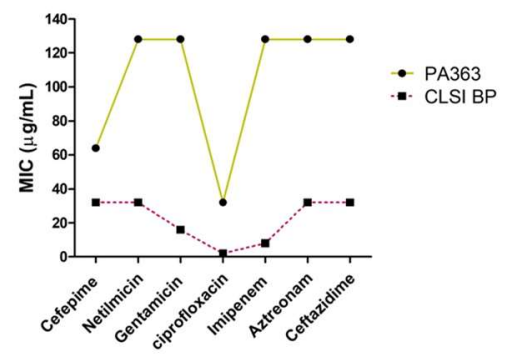

E

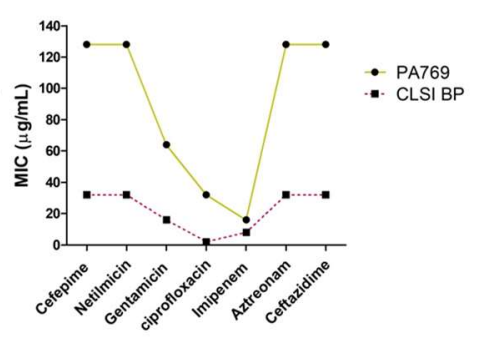

H

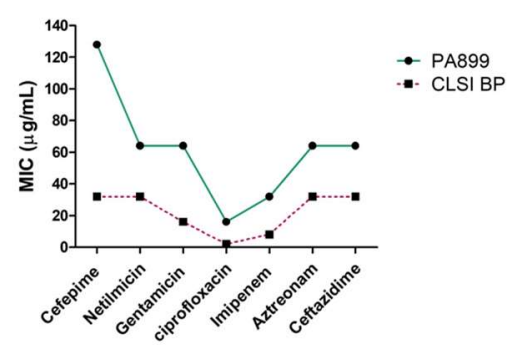

C

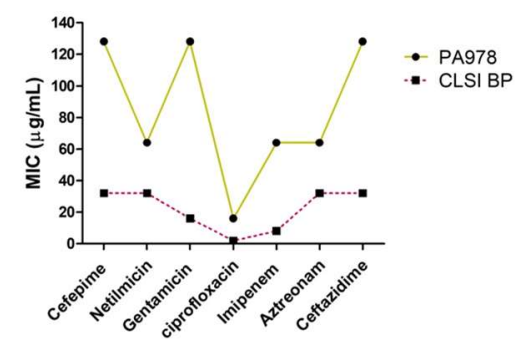

$\mathbf{F}$

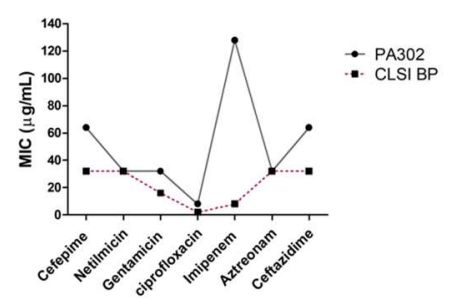

I

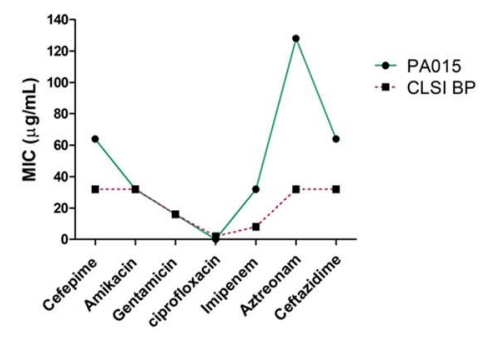

Figure 5: MIC of metallo-beta-lactamase-producing pigmented and non-pigmented $\boldsymbol{P}$. aeruginosa. Comparative MIC pattern for tested antibiotics according to Clinical \& Laboratory Standards Institute guidelines for yellow pigment (PA103, PA363, PA978, PA786, PA769), (A-E); Non pigmented (PA302, PA407), (F, G); and Green pigmented producing strains (PA899, PA015) (H, I). 